\title{
Au commencement était la vie?
}

\section{Bruno Kesseli}

Dr méd. et lic. phil., rédacteur en chef
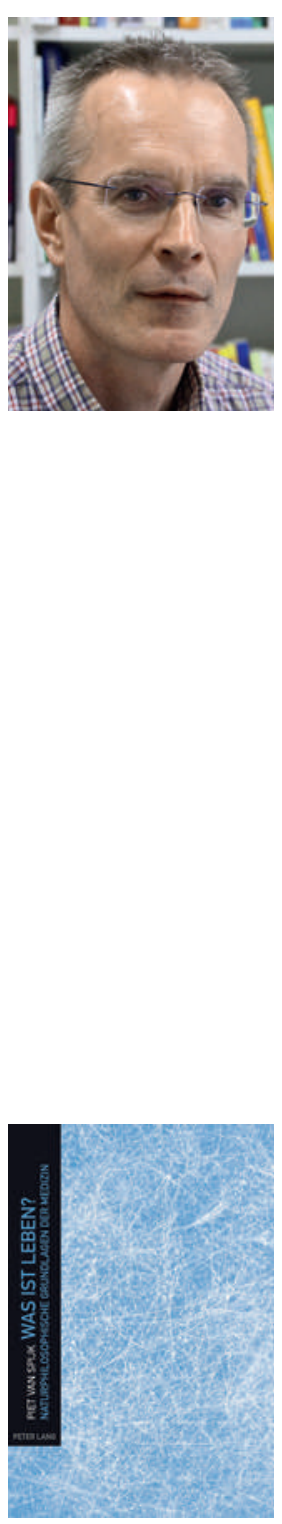

Piet van Spijk Was ist Leben?

Naturphilosophische Grundlagen der Medizin Berne: Peter Lang; 2014. 297 pages. 87 CHF. ISBN 978-3-0343-1321-6

bkesseli[at]emh.ch
Des souvenirs durables que j'ai gardés de mes études de médecine, les premières paroles du professeur Jeremias Kägi à nous autres étudiants de deuxième année restent l'un des plus marquants. Ce jour-là, celui qui était à l'époque directeur de l'Institut de biochimie de l'Université de Zurich a commencé sa leçon en disant à peu près ceci: «La biochimie étudie les processus de la vie. Ce qu'est la vie au juste - c'est bien connu - personne ne le sait.»

Pour inhabituelle qu'elle fût, cette entrée en matière n'a certainement pas nui à l'image du professeur auprès de ses étudiants - bien au contraire. Si ces paroles m'ont marqué, c'est aussi parce que venant de grandes pointures académiques, des aveux aussi francs sur les frontières de la connaissance scientifique ont valeur de rareté. A quelques variantes près, la réponse standard à nos questions est en effet toujours la même: «Tous les détails ne sont pas encore élucidés», sur un ton qui se veut rassurant, l'air de dire: «Patience, ça ne saurait tarder.»

Loin de moi l'idée de vouloir relativiser les dernières avancées fantastiques des sciences de la vie et des biotechnologies, dont nous profitons tous dans notre vie quotidienne, mais après avoir accumulé sur le vivant un niveau presque inquiétant de connaissances détaillées, analysé la vie sous de nombreuses formes et être devenus capables de la domestiquer à loisir, force nous est de constater que les grandes questions demeurent sans réponse. D’où vient la vie? Comment est-elle née? Est-il possible de transformer la matière morte en quelque chose de vivant? Il nous faut une réponse sans équivoque à ces questions avant de pouvoir prétendre élucider le mystère de la vie. Y parviendra-t-on un jour? Rien n'est moins sûr.

Notre expérience quotidienne nous enseigne que la vie ne peut naître que de la vie et que tout être vivant finit par mourir. Personne que je connaisse n'a encore observé le phénomène inverse d'une structure morte qui prendrait vie. Or, la science naturelle pense devoir postuler que c'est précisément ce qui se produit si les bonnes conditions sont réunies. A défaut, elle devrait introduire dans ses équations des inconnues et des forces qui n'ont rien à y faire, mais que l'on trouve dans des écrits religieux relatant des phénomènes proches de la science-fiction. A l'exemple de la Bible qui décrit la résurrection de Jésus - c'est bientôt Pâques - ou le réveil de Lazare du monde des morts, alors que sa dépouille pourrissante dégageait déjà une odeur cadavérique. Avec le bon vieux hasard qui, d'un claquement de doigts, éveille à la vie des structures auto-organisées de bicouches lipidiques, d'acides aminés et d'acides nucléiques baignant dans la soupe primitive, on est quand même en terrain plus sûr, n'est-ce pas?

Outre les sciences naturelles et la religion, la philosophie aussi mène une réflexion importante sur le "problème de la vie». Certains philosophes sont aussi méde-

Avec son livre, Piet van Spijk a jeté dans la mare un pavé qui devrait faire des vagues.

Il sera intéressant de voir si elles font bouger les choses.

cins (et vice-versa), le corps médical est donc bien placé pour contribuer de manière substantielle à ce débat; après tout, le médecin se consacre quotidiennement aux soins du plus complexe de tous les êtres vivants. Piet van Spijk, philosophe et médecin interniste exerçant à Lucerne, le fait avec brio. Son livre Was ist Leben? Naturphilosophische Grundlagen der Medizin (Qu'est-ce que la vie? Fondements philosophiques de la médecine en tant que science naturelle), paru en 2014, traite des questions évoquées et de bien d'autres aspects du phénomène qu'est la vie. C'est un ouvrage exigeant, pas un cours d'introduction en 48 heures pour philosophes de salon. C'est aussi une lecture particulièrement recommandée aux médecins, vu qu'en plus des aspects théoriques, le livre traite aussi des conséquences pratiques et importantes pour la médecine d'une vision du monde qui, sans être fondamentalement nouvelle, a tendance à rester dans l'ombre. La vie, et non la mort, est le principe primordial: telle est l'hypothèse centrale qui offre une solution élégante à quelques problèmes, notamment en rapport avec l'apparition de la vie. Non pas que l'adage de mon professeur soit désormais dépassé - le débat sur la nature de la vie n'est sans doute pas près de se clore. Mais avec son livre, Piet van Spijk a en quelque sorte jeté dans la mare un pavé qui devrait faire des vagues. Il sera intéressant de voir si elles font bouger les choses ou si elles se dissipent dans l'océan de l'esprit du temps. 